\title{
Influence of Air Annealing and Gamma Ray Irradiation on the Optical Properties of $\mathrm{Cl}_{16} \mathrm{FePc}$ Thin Films
}

\author{
RAJI KOSHY AND C. S. MENON \\ School of Pure and Applied Physics, \\ Mahatma Gandhi University, Kottayam, Kerala 686560, India \\ rajirose23@yahoo.com
}

Received 04 October 2011; Accepted 07 March 2012

\begin{abstract}
Metal phthalocyanines are one of the most promising candidates to be used in the fabrication of such devices. Among various phthalocyanines, Iron Hexadecachloro Phthalocyanine $\left(\mathrm{Cl}_{16} \mathrm{FePc}\right)$ has received less attention. Basic characteristics of $\mathrm{Cl}_{16} \mathrm{FePc}$ are not reported in literature. Hexadecacholoro phthalocyanines have attracted interest as possible n-type organic semiconductor with high electron mobility and good stability characteristics. In the present work we investigate the optical band gap of the $\mathrm{Cl}_{16} \mathrm{FePc}$ thin films from the optical absorption spectrum as a function of air annealing temperatures and their suitability for the fabrication of molecular electronic devices. Some optical properties of the samples were studied as a function of $\gamma$-radiation doses also. Optical transition is found to be of direct type and optical band gaps are determined by analyzing the absorption spectrum. Vacuum sublimed thin films of Iron hexadecachloro phthalocyanine were prepared at room temperature onto glass substrates at a base pressure of $10^{-5}$ Torr on precleaned glass substrates using Hind Hivac $12 \mathrm{~A} 4$ coating plant. The optical energy band gap Eg were calculated. The mechanism of optical absorption follow the rule of direct transition. In the present paper we also report refractive index, real and imaginary parts of optical dielectric constant etc. from the reflectance measurements.
\end{abstract}

Keywords: Iron hexadecachloro phthalocyanine, Thin films, Optical properties, Air annealing, $\gamma$-irradiation.

\section{Introduction}

Thin films of organic semiconducting molecules continue to attract a significant degree of attention due to their optical and electronic properties and possibility to modify these are changing specific functionalities of the molecules. Phtalocyanines are model systems of this interesting feature, since by changing the central atom, the gap in the electronic spectrum can be modified ${ }^{1}$. These materials have high thermal stability, strong colours and negligible vapour pressure at room temperature. The study of the electrical conductivity and the 
nonlinear optical properties of phtalocyanine thin films are of great interest for use in molecular electronics, non - linear optics, electro chromic devices, optical data storage, sensors, solar cells, light emitting diodes and static induction transistors ${ }^{2-5}$. Phtalocyanines can be sublimed without decomposition to form high quality thin films by the thermal evaporation technique. The stability of these films, however at high temperature and high radiation environment is still a matter of research. Physical properties of phtalocyanines are strongly influenced by various factors such as the evaporation rate, substrate temperature, film thickness and post deposition annealing ${ }^{6}$. Radiation processing is an expanding technology with numerous applications in the field of health care products sterilization, sewage and hospital waste treatments, polymer modifications and food processing. Irradiation of thin films with high energy radiations like $\gamma$ - rays is expected to affect their physical properties. The halogenated pcs exhibit remarkable physical properties and thermal stability over a large temperature range compared to unhalogenated pcs ${ }^{7}$. The band gap determination is particularly useful to detect the impurity levels and defects when working with new compound semiconductors since radiative transitions in semiconductors involve localized defect levels. Electrons excited from valance band to the conduction band with the absorption of energy equal to the band gap of the material. The sudden fall in the absorption coefficient on the high energy side of the absorption spectra leads to the band edge in semiconductor systems. The study of irradiated samples enhances the efficiency improvement in its applicability in a radiation environment and is also important in obtaining basic information on vacancies, defects, and their interaction with impurities ${ }^{8,9}$. In this work, we have made a detailed investigation on the effect of air annealing and $\gamma-$ ray irradiation on the optical properties of vacuum deposited $\mathrm{Cl}_{16} \mathrm{FePc}$ thin films. This article brings out the results of the optical band gap, refractive index and dielectric constants, from the absorption co-efficient and extinction co-efficient of $\mathrm{Cl}_{16} \mathrm{FePc}$ thin films, which is essential for the fabrication of electronic devices. Optical constants of thin films are influenced by various preparation parameters and post-deposition heat treatment given to the films. Results show that $\mathrm{Cl}_{16} \mathrm{FePc}$ is highly stable against heat, high energy radiations and atmospheric air. In addition hexadecahalogenated phthalocyanines attracted interest as a possible n-type organic semiconductor with high electron mobility and good stability characteristics ${ }^{10,11}$. This suggests that hexadecahalogenated phthalocyanines are useful to develop molecular electronic devices. Figure 1 shows the molecular structure of $\mathrm{Cl}_{16} \mathrm{FePc}_{\text {. }}$.

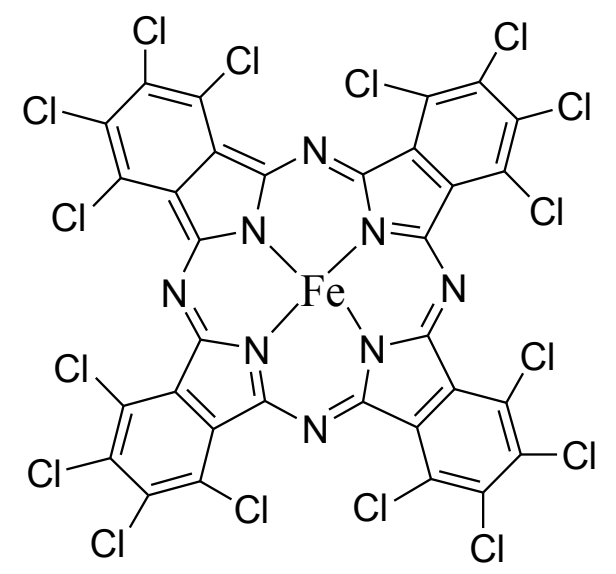

Figure 1. The molecular structure of $\mathrm{Cl}_{16} \mathrm{FePc}$. 


\section{Materials and Methods}

Iron hexadecachloro phthalocyanine thin films are deposited at room temperature at a base pressure of $10^{-6}$ Torr on pre-cleaned glass substrates using the Hind Hivac 12 A4 coating plant by thermal evaporation. Molybdenum boat is used as the resistive heating element for coating. Substrates are placed at a distance of $12 \mathrm{~cm}$ from the boat and deposition rate is controlled to $12 \mathrm{~nm} / \mathrm{min}$. The thickness of the films is measured using Dektac thickness profilometer and is cross checked by Tolansky's multiple beam interference technique ${ }^{12}$. Thin films deposited at room temperature are annealed at temperatures 373,423 and $473 \mathrm{~K}$ in air for 1 hour using a furnace attached with a digital temperature controller cum recorder. UV-Vis absorption spectra are recorded using Shimadzu 160A spectrophotometer. Reflection spectrum is recorded by Jasco V-570 spectrophotometer. $\mathrm{Cl}_{16} \mathrm{FePc}$ thin films were irradiated with ${ }_{\text {Co }}^{60} \gamma$ source. Radiation doses were controlled through the exposure time.

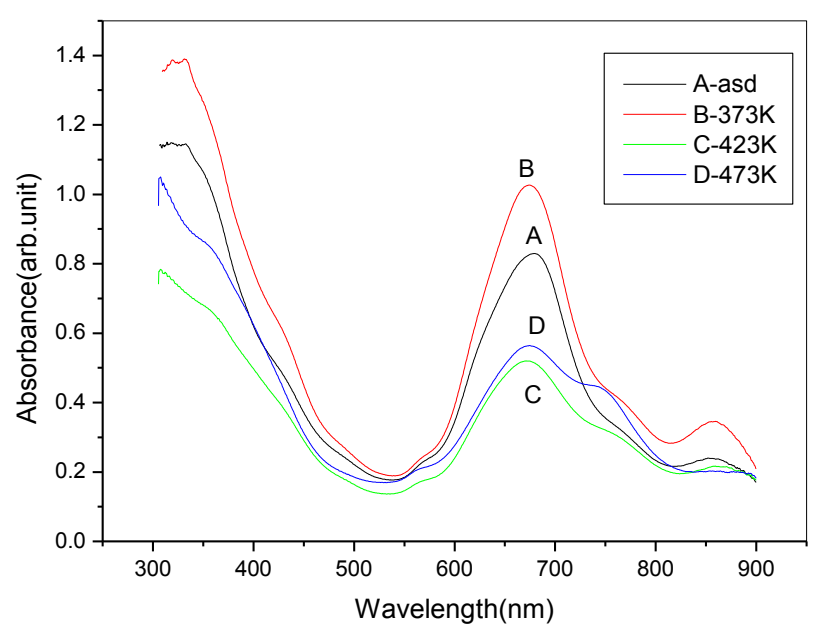

Figure 2. Optical absorption spectra of $\mathrm{Cl}_{16} \mathrm{FePc}$ thin films of as-deposited form and annealed at 373,423 and $473 \mathrm{~K}$ in air.

\section{Results and Discussions}

Optical analysis of $\mathrm{Cl}_{16} \mathrm{FePc}$ thin films is done to get the absorption co-efficient and extinction coefficient, refractive index and their dependence on wavelength of the incident radiation. The study of optical absorption is a useful method for investigating the induced transition and providing information about the band structure in the materials. Optical studies are done to determine the energy band gap and the effect of annealing on the band gap. The principle of this technique is that photons with energies higher than the band gap energy will be absorbed and electrons are excited from the valance band to the conduction band with the absorption of energy equal to the band gap of the material. The transition type is also important to be defined for the material characterization. The optical absorption spectra of films annealed in air at temperatures 373, 423, 473K and as- deposited form over a wavelength range of $300-900 \mathrm{~nm}$ are shown in Figure 2. The thickness of the films are $350 \pm 5 \mathrm{~nm}$. 


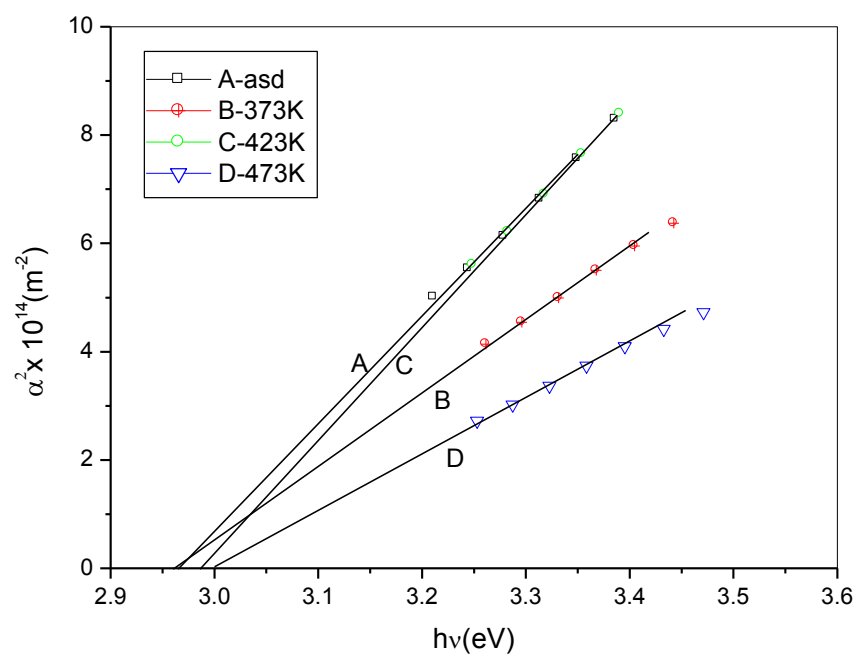

Figure 3. Plot of $\alpha^{2}$ versus photon energy hv $\mathrm{Cl}_{16} \mathrm{FePc}$ thin films as-deposited and annealed at 373,423 and $473 \mathrm{~K}$ in air.

It has been suggested that the UV-Vis spectrum obtained for pcs originates from the molecular orbitals within the aromatic $18 \pi$ electron system and from overlapping on the central metal atom ${ }^{13}$. The $\pi$ and $\pi^{*}$ molecular orbitals are the highest occupied molecular orbital (HOMO) and the lowest unoccupied molecular orbital (LUMO) respectively in terms of molecular physics. They are also the valance and conduction bands of semiconductor physics. Both $\mathrm{Q}$ and $\mathrm{B}$ bands are the direct electronic transitions arise from $\pi-\pi^{*}$ transitions, which gives the absorption edge. To obtain information about direct or indirect interband transition, the fundamental absorption edge is analyzed within the frame work of one electron theory of Bardeen et.al. This theory has been used to analyze the absorption edge data of molecular solids such as phthalocyanine ${ }^{14}$ and the absorption coefficient is calculated from the data. The coefficient $\alpha$ is related to the photon energy h $v$ by the relation

$$
\alpha=\alpha_{0}\left(h v-E_{g}\right)^{n},
$$

where $n=1 / 2$ for direct allowed transitions, Eg being the optical band gap and $\alpha_{0}$ a constant. Plot of $\alpha^{2} v s$ h $v$ near the absorption edge for as-deposited, annealed at 323,423 and 473K of $\mathrm{Cl}_{16} \mathrm{PcFe}$ thin films are as shown above in Figure 3.

A satisfactory fit is obtained for $\alpha^{2}$ vs $h v$ indicating the presence of direct band gap. The optical band gap is determined as $2.97 \mathrm{eV}$, from the $\alpha^{2} \mathrm{vs} \mathrm{h} v$ graph given in Figure 3.

The optical parameters of $\mathrm{Cl} 16 \mathrm{FePc}$ thin films can be determined from the reflectance spectra. The reflectance spectrum of Cl16FePc thin film over the wavelength range 200nm$900 \mathrm{~nm}$ for the as deposited film is given in Figure 4. 


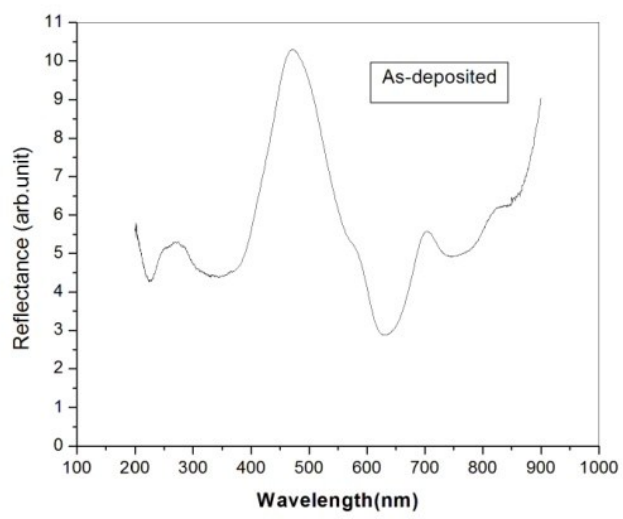

Figure 4. Reflectance versus wavelength for as-deposited $\mathrm{Cl}_{16} \mathrm{FePc}$ thin film.

The extinction coefficient $\mathrm{k}$ is calculated using the equation.

$$
\mathrm{k}=\frac{\alpha \lambda}{4 \pi}
$$

where $\alpha$ is the absorption coefficient and $\lambda$ is the wavelength. The reflectivity of an absorbing medium of indices $\mathrm{n}$ and $\mathrm{k}$ in air for normal incidence is given by

$$
\mathrm{R}=\frac{(n-1)^{2}+k^{2}}{(n+1)^{2}+k^{2}}
$$

Thus by knowing the $\mathrm{R}$ and $\mathrm{k}, \mathrm{n}$ is calculated.

Table 1. band gaps obtained for $\mathrm{Cl}_{16} \mathrm{FePc}$ thin films annealed in air at various temperatures, as-deposited and irradiated with various dosages.

\begin{tabular}{|l|l|}
\hline $\begin{array}{l}\text { As-deposited, } \\
\text { Annealing Temperatures } \\
\text { Gamma ray Doses }\end{array}$ & Band Gap \\
\hline As-deposited & 2.97 \\
\hline $373 \mathrm{~K}$ & 2.96 \\
\hline $423 \mathrm{~K}$ & 2.98 \\
\hline $473 \mathrm{~K}$ & 3.00 \\
\hline $400 \mathrm{~Gy}$ & 2.62 \\
\hline $800 \mathrm{~Gy}$ & 2.62 \\
\hline $1200 \mathrm{~Gy}$ & 2.61 \\
\hline
\end{tabular}

The optical properties of the medium are characterized by a complex refractive index $(\mathrm{N}=\mathrm{n}$-ik) and complex dielectric constant. The real and imaginary part of the optical dielectric constant $\varepsilon_{1}$ and $\varepsilon_{2}$ are calculated from the data obtained from the reflection spectrum. The real part $\varepsilon_{1}$ generally relates to dispersion, while the imaginary part $\varepsilon_{2}$ provides measure of the dissipative rate of the wave in the material. The dependence of $\varepsilon_{1}$ and $\varepsilon_{2}$ on photon 
energy and dependence of $\mathrm{n}$ and $\mathrm{k}$ on photon energy for $\mathrm{Cl}_{16} \mathrm{FePc}$ thin films of as-deposited form are plotted in the Figure $5 \& 6$.

The refractive index $\mathrm{n}$ has a maximum value of 1.94 at $2.63 \mathrm{eV}$ for the $\mathrm{Cl} 16 \mathrm{FePc}$ thin film. The extinction coefficient $\mathrm{k}$ has a maximum value of 0.30 at $1.81 \mathrm{eV}$. The real part $\varepsilon 1$ shows a maximum value of 3.76 at $2.63 \mathrm{eV}$ while imaginary part $\varepsilon 2$ has a maximum value of 0.94 at $1.78 \mathrm{eV}$. It is observed that these parameters vary with photon energy.

The intersept on the energy axis, as shown in the above Figure gives the band gap Eg of the material. The band gap of unirradiated and irradiated samples are $2.97 \mathrm{eV}$ and $2.62 \mathrm{eV}$ respectively. It is found that optical band gap decreases with increase of gamma dosage. This is attributed to the increase in disorder due to the presence of structural defects, which in turn increase the width of localized states and hence a decrease in optical band gap ${ }^{15,16}$. The absorption at lower energy side is related to singlet exactions and has been confirmed for many other phthalocyanines ${ }^{17}$.

The band gaps obtained for $\mathrm{Cl}_{16} \mathrm{FePc}$ thin films annealed in air at different temperatures, asdeposited and irradiated with various dosages are given in Table1.

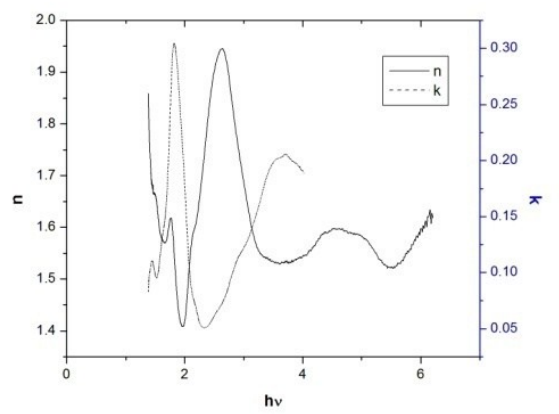

Figure 5. Plot of refractive index $n$ and extinction coefficient $\mathrm{k}$ versus photon energy for as-deposited $\mathrm{Cl}_{16} \mathrm{FePc}$ Thin Film.

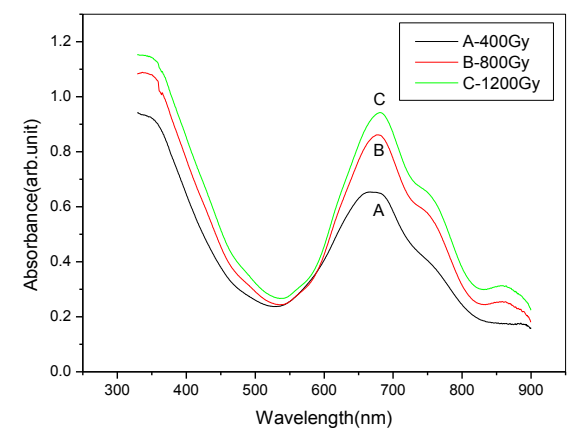

Figure 7. optical absorption spectra of $\mathrm{Cl}_{16} \mathrm{FePc}$ thin films irradiated with various dosages.

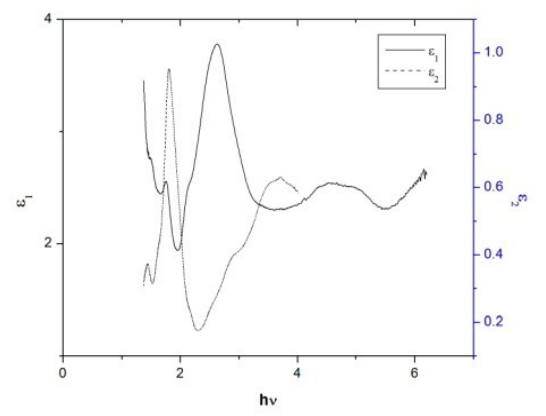

Figure 6. Plot of real and imaginary parts of the dielectric constant versus photon energy for as-deposited $\mathrm{Cl}_{16} \mathrm{FePc}$ thin film.

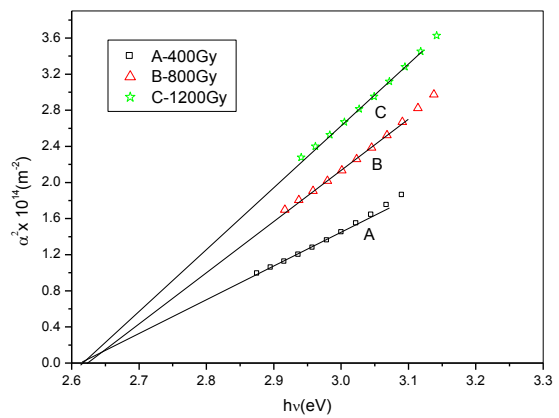

Figure 8. Plot of $\alpha^{2}$ versus photon energy $\mathrm{h} v$ for $\gamma$ - irradiated $\mathrm{Cl}_{16} \mathrm{FePc}$ thin $\gamma$-radiation films at different dosages. 


\section{Conclusion}

The optical absorption and particularly the absorption band edge is a good method for studying optically induced transition and gives the structure and optical energy gap in thin films. The basic optical properties of as - deposited, air annealed and gamma ray irradiated at various dosages of $\mathrm{Cl}_{16} \mathrm{FePc}$ thin films have been studied. The fundamental energyband gap is calculated corresponding to the band edge. The absorption spectrum of all samples show two prominent peaks, the one at the shorter wavelength region $321 \mathrm{~nm}$ resulting from the $\mathrm{B}$ (Soret) band and that at the longer wavelength region $680 \mathrm{~nm}$ resulting from the $\mathrm{Q}$ band. The dependence of absorption coefficient $\alpha$ on photon energy h $v$ is determined for $\mathrm{Cl}_{16} \mathrm{FePc}$ thin films of thickness $350 \pm 5 \mathrm{~nm}$ under different heat treatments. A direct allowed transition is observed. The optical band gap energy is $2.97 \pm 0.01 \mathrm{eV}$ and it does not show much variation under different heat treatments. The band gap of unirradiated and irradiated samples are $2.97 \mathrm{eV}$ and $2.62 \mathrm{eV}$ respectively. It is found that optical band gap decreases with increase of gamma dosage. This is attributed to the increase in disorder due to the presence of structural defects, which in turn increase the width of localized states and hence a decrease in optical band gap. This can help the fabrication of molecular electronic devices using $\mathrm{Cl}_{16} \mathrm{FePc}$ thin films. Fundamental optical parameters have been determined from both absorption and reflection data. In future $\mathrm{Cl}_{16} \mathrm{FePc}$ thin films can be used in fabrication of $\mathrm{p}-\mathrm{n}$ junction diodes.

\section{References}

1. D.Schelettwein, in : H.S.Nalwa (Ed.), Super molecular Photosensitive and Electro active Materials, Academic Press, San Diego, 2001.

2. Y.Qiu, Y.Gao, P.Wei, L.Wang, Appl.Phys.Lett, 2002, 80, 2628.

3. J.Zhang, J.Wang, H.Wang, D.Yane, Appl.Phys. Lett., 2004, 84, 142.

4. P.Perumans, S.R.Forrest. Appl.Phys.Lett, 2001, 79, 126.

5. C.M.Joseph, C.S.Menon, Mater. Lett., 2002, 52, 220.

6. Ambily.S, Menon.C.S, Thin Solid Films, 1999, 347, 284.

7. A.Napier, R.A.Collins, Phys.Stat.Sol (A), 1994, 144, 91.

8. Arshak.K., Korostynska.O, Fahim.F, Sensors, 2003, 3, 176.

9. Abu.El-Fadl.A, El-Maghraby.E.M, Mohamad.G.A, Cryst.Res.Technol., 2004, 39,143.

10. Z.Bao, A.J.Lovinger, J.Brown, J.Am.Chem.Soc. 1998, 120, 207.

11. K.W.Hipps, Louis Schudiero, Dan.E.Barlow, and Manning.P.Cook, Jr.Amer.Chem.Soc. February 2002.

12. L.I.Maissel, R.Glang, Handbook of Thin Film Technology, Mc Graw-Hill, New York, 1985

13. E.A.Ough, J.M.Stillman, Can.J.Chem., 1993, 71, 1891.

14. S.Ambily, C.S.Menon, Sol.Stat.Commun, 1995, 94,485.

15. N. F. Mott, E. A. Davis, Electronic Process in Non-Crystalline Materials, $2^{\text {nd }}$ ed. Claendon Press, Oxford (1979).

16. A. Sussman, J. Appl.Phys., 1967, 38, 2738.

17. Lucia. E.A \& Verderame. F.D, J Chem Phys , 1968, 48, 2674. 


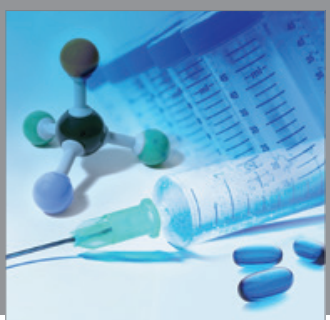

International Journal of

Medicinal Chemistry

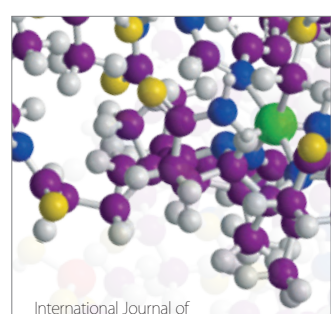

Carbohydrate Chemistry

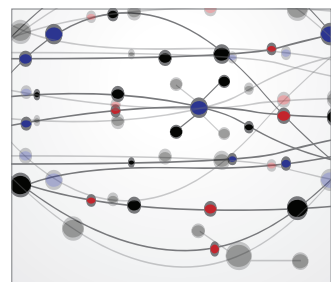

The Scientific World Journal
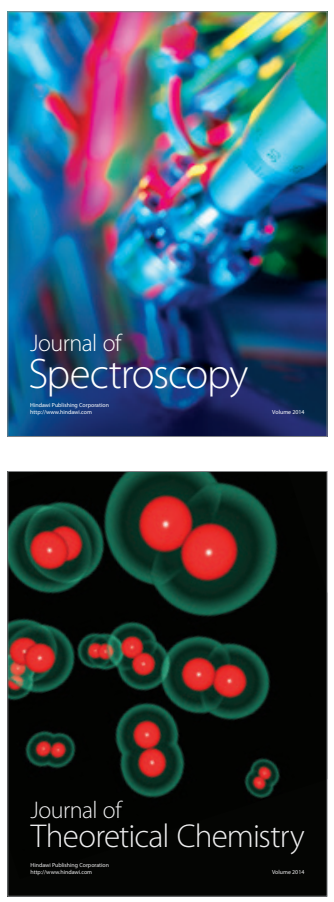
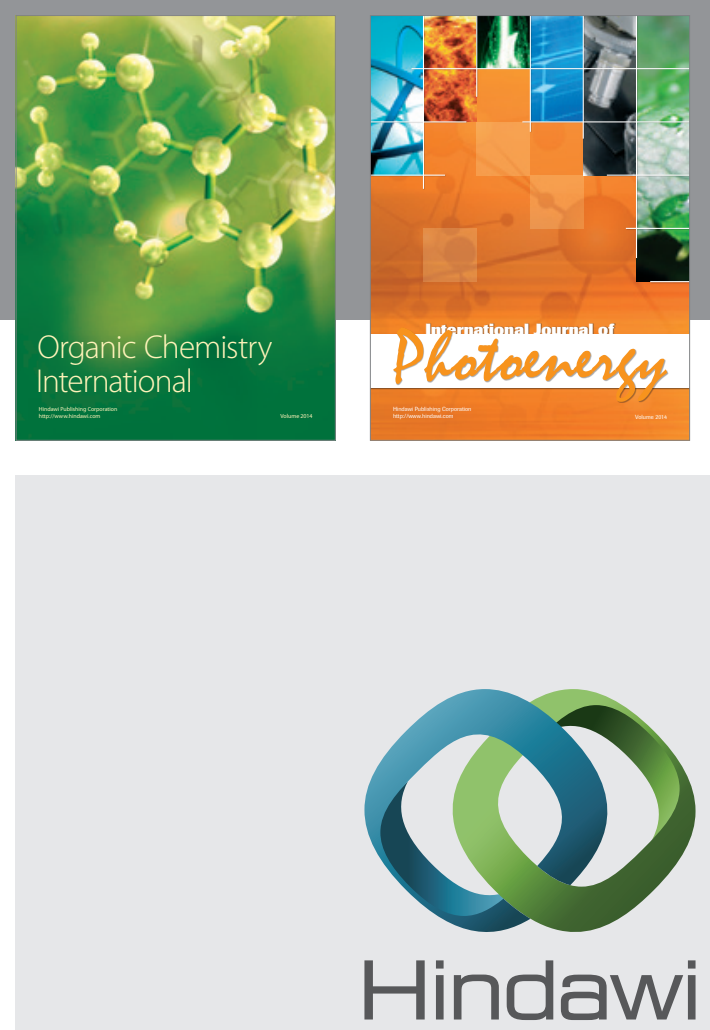

Submit your manuscripts at

http://www.hindawi.com
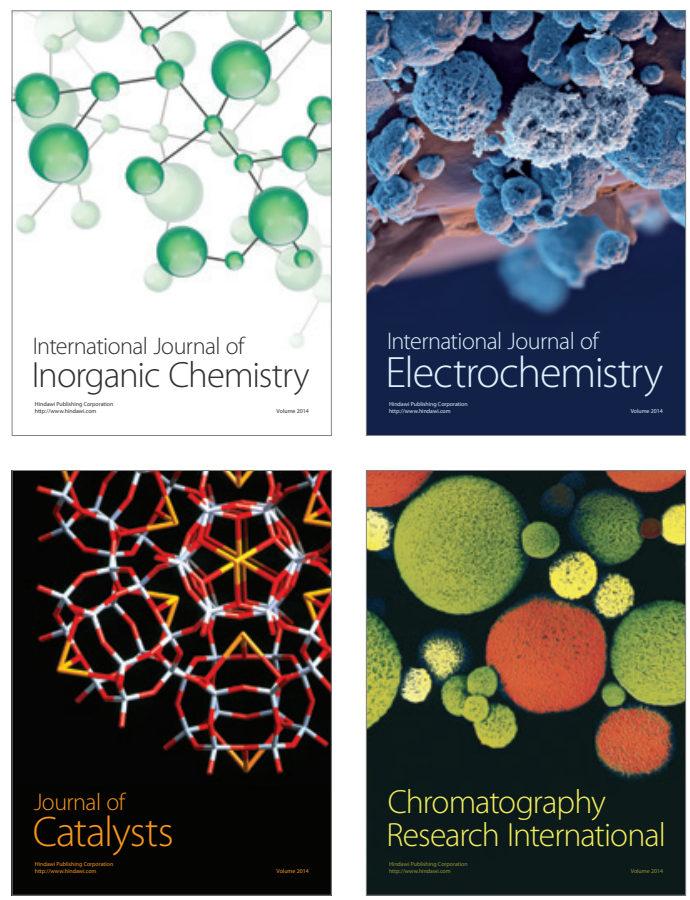
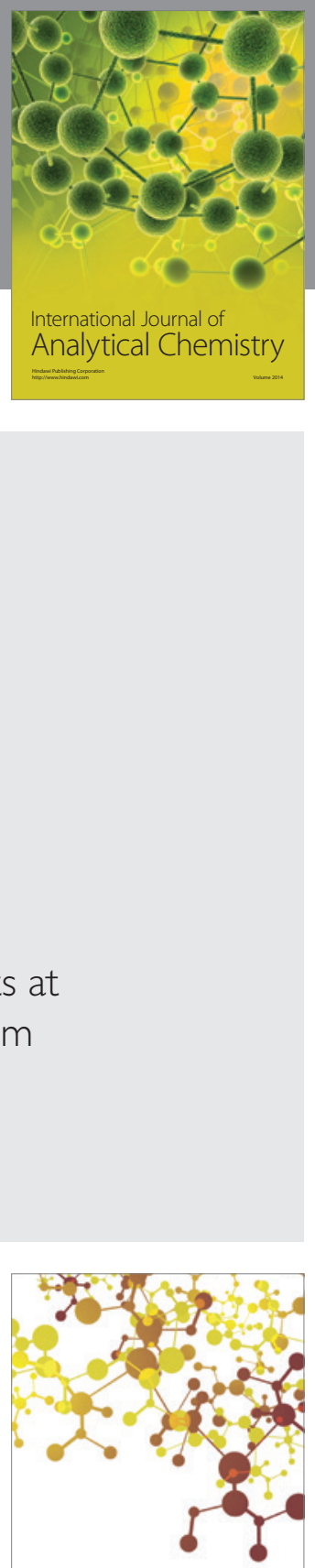

Journal of

Applied Chemistry
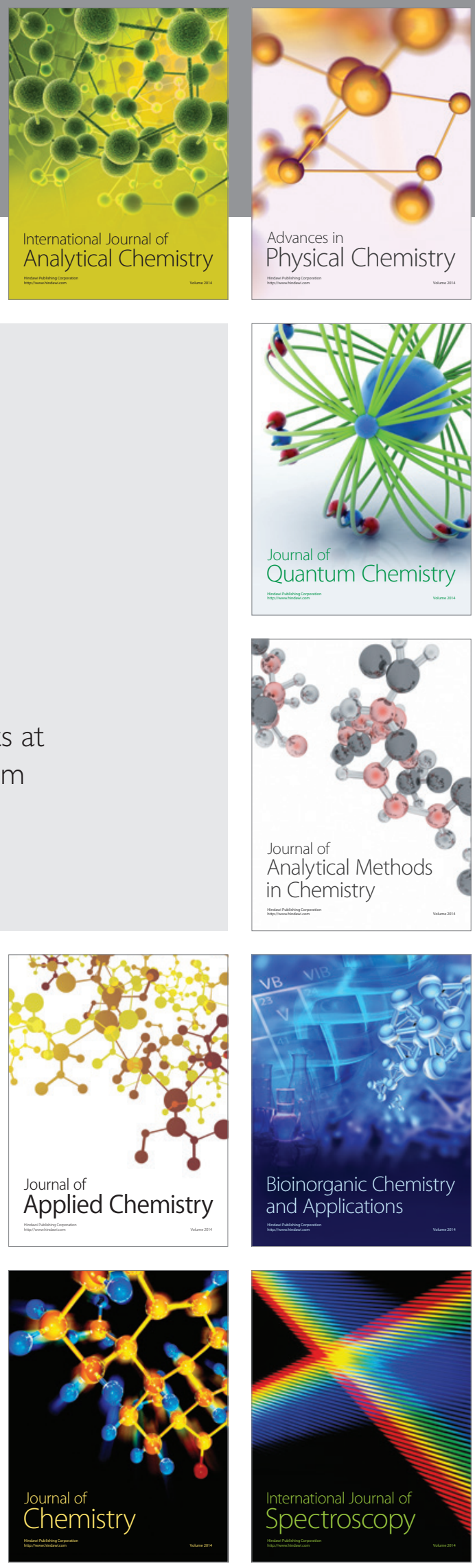